\title{
Math-Net.Ru
}

П. А. Головач, Ф. В. Фомин, Суммарная величина вершинного разделения и профиль графов, Дискрет. матем., 1998, том 10, выпуск 1, 87-94

DOI: https://doi.org/10.4213/dm410

Использование Общероссийского математического портала Math-Net.Ru подразумевает, что вы прочитали и согласны с пользовательским соглашением http://www.mathnet.ru/rus/agreement

Параметры загрузки:

IP : 54.197 .130 .99

26 апреля 2023 г., 12:32:19

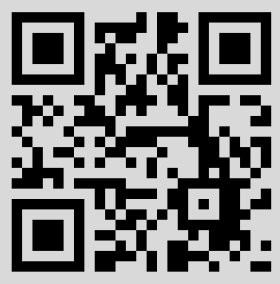




\title{
Аискретная математика \\ том 10 выпуск $1 * 1998$
}

УДК 519.717

\section{Суммарная величина вершинного разделения и профиль графов}

\author{
(C) 1998 г. П. А. Головач, Ф. В. Фомин
}

\begin{abstract}
Рассматриваются два инварианта графов, определяемые через оптимальные (по различным критериям) нумерации вершин. Это суммарная величина вершинного разделения и профиль графов. Доказывается, что эти два инварианта равны. Приводится ряд свойств профиля графов, полученных с помощью равенства этих инвариантов.

Работа выполнена при поддержке Российского фонда фундаментальных исследований, проект 96-02-00285
\end{abstract}

\section{1. Введение}

В настоящее время известна и активно изучается серия инвариантов графов, определяемых с помощью оптимальных (по различным критериям) нумераций вершин. Это, в частности, такие характеристики графов, как ширина ленты, ширина разреза, величина вершинного разделения и некоторые другие. $\mathrm{C}$ данными инвариантами тесно связан ряд инвариантов, определяемых через иные задачи оптимизации. $\mathrm{K}$ их числу относятся такие характеристики графов, как поисковое число, путевая ширина, древесная ширина графа. Внимание ко всем этим величинам объясняется как теоретическим интересом, так и тем, что соответствующие им задачи оптимизации естественным образом возникают в приложениях. Более подробную информацию о данных инвариантах можно получить, например, в обзорах $[1,2,3]$.

В работе [4] один из авторов данной статьи ввел в рассмотрение еще один инвариант графов, определяемый с помощью оптимальных нумераций вершин, названный суммарной величиной вершинного разделения графа. Эта характеристика графа близка к уже довольно хорошо исследованному инварианту, величине вершинного разделения (см. $[2,5])$, и находится к нему в том же отношении, в каком находится суммарная ширина ленты графа к ширине ленты, то есть отличается лишь нормой, по которой проводится оптимизация. В [4] был получен ряд результатов, касающихся суммарной величины вершинного разделения. В частности, был рассмотрен вопрос о характеризации данного инварианта с помощью графов интервалов, определена сложность вычисления суммарной величины вершинного разделения. Позднее, другим автором настоящей статьи было указано, что суммарная величина вершинного разделения совпадает с уже известным инвариантом, а именно, с профилем графов. 
В нашей работе изложено доказательство равенства между суммарной величиной вершинного разделения и профилем графов. Кроме того, приводится ряд свойств профиля графов, полученных с помощью равенства этих инвариантов.

\section{2. Основные определения}

В этом разделе вводятся основные понятия, необходимые для изложения результатов.

Пусть $G$ - граф с $n$ вершинами. Отметим сразу, что в данной работе будут рассматриваться только простые графы.

Через $V(G)$ (или просто через $V$, если понятно, о каком графе идет речь) мы будем обозначать множество вершин графа $G$, а через $E(G)$ (или просто $E$ ) множество ребер.

Если $U \subseteq V$, то положим

$$
\partial U=\{u: u \in U \text { и существует вершина } v \in V \backslash U \text {, смежная } u\} \text {. }
$$

Нумерацией вершин графа $G$ называется взаимно однозначное отображение

$$
f: V \rightarrow\{1, \ldots, n\} .
$$

Пусть $f$ - нумерация вершин графа $G$. Положим

$$
S_{i}(G, f)=\{v: v \in V, f(v) \leqslant i\}, \quad i=1, \ldots, n .
$$

Величиной вершинного разделения (см. [5]) графа $G$ называется величина

$$
\operatorname{vs}(G)=\min _{f} \max _{1 \leqslant i \leqslant n-1}\left|\partial S_{i}(G, f)\right|
$$

где минимум берется по всевозможным нумерациям вершин графа.

Назовем суммарной величиной вершинного разделения графа $G$ величину

$$
\mathrm{vs}_{\mathrm{sum}}(G)=\min _{f} \sum_{i=1}^{n-1}\left|\partial S_{i}(G, f)\right| .
$$

Заметим (см. [1]), что шириной ленты графа $G$ называется величина

$$
b(G)=\min _{f} \max _{(u, v) \in E}|f(u)-f(v)|
$$

а суммарной шириной ленты величина

$$
b_{\text {sum }}(G)=\min _{f} \sum_{(u, v) \in E}|f(u)-f(v)| .
$$

Таким образом, суммарная величина вершинного разделения находится в том же отношении к величине вершинного разделения, в каком суммарная ширина ленты находится к ширине ленты.

Для того, чтобы определить профиль графа, введем следующее обозначение. Если $u, v$ - вершины графа, то будем писать $u \cong v$ в том случае, если либо $u=v$, либо вершины $u$ и $v$ являются смежными. 
Профилем графа $G$ (см. [1]) называется величина

$$
p(G)=\min _{f} \sum_{u \in V}(f(u)-\min \{f(v): v \in V, v \cong u\}),
$$

где, как и выше, минимум берется по всевозможным нумерациям вершин графа.

\section{3. Суммарная величина вершинного разделения и профиль графов}

В этом параграфе будет показано, что для любого графа $G$

$$
\operatorname{vs}_{\text {sum }}(G)=p(G) \text {. }
$$

Начнем с доказательства одного вспомогательного утверждения. Пусть $G$ - произвольный граф. Положим

$$
p^{*}(G)=\min _{f} \sum_{u \in V}(\max \{f(v): v \in V, v \cong u\}-f(u)) .
$$

Лемма 1. Для любого графа $G$

$$
p(G)=p^{*}(G)
$$

Доказательство. Докажем, что $p(G) \geqslant p^{*}(G)$.

Пусть граф $G$ имеет $n$ вершин и пусть $f$ - оптимальная (для профиля) нумерация вершин графа, то есть

$$
p(G)=\sum_{u \in V}(f(u)-\min \{f(v): v \in V, v \cong u\}) .
$$

Введем в рассмотрение нумерацию $f^{*}$, определяемую равенством

$$
f^{*}(u)=n+1-f(u)
$$

для каждой вершины $u \in V$.

Нетрудно убедиться, что для всякой вершины $u \in V$ выполняется равенство

$$
\max \left\{f^{*}(v): v \cong u\right\}=n+1-\min \{f(v): v \in V, v \cong u\}
$$

а потому

$$
\max \left\{f^{*}(v): v \cong u\right\}-f^{*}(u)=f(u)-\min \{f(v): v \in V, v \cong u\}
$$

Суммируя правую и левую части этого равенства по всем $u \in V(G)$, и учитывая, что $f$ - оптимальная нумерация, получаем искомое неравенство $p(G) \geqslant p^{*}(G)$.

Неравенство $p(G) \leqslant p^{*}(G)$ доказывается аналогичнс.

Лемма доказана.

Теперь докажем основное утверждение этого раздела. 
Теорема 1. Для любого графа $G$

$$
p(G)=\operatorname{vs}_{\mathrm{sum}}(G) .
$$

Доказательство. В силу леммы 1 для доказательства теоремы достаточно показать, что

$$
p^{*}(G)=\mathrm{vs}_{\mathrm{sum}}(G) .
$$

Пусть граф $G$ имеет $n$ вершин, и пусть $f$ - произвольная нумерация вершин этого графа. Докажем, что

$$
\sum_{u \in V}(\max \{f(v): v \in V, v \cong u\}-f(u))=\sum_{i=1}^{n-1}\left|\partial S_{i}(G, f)\right| .
$$

Для этого заметим, что вершина $u$ принадлежит множеству $\partial S_{i}(G, f)$ тогда и только тогда, когда

$$
f(u) \leqslant i<\max \{f(v): v \in V, v \cong u\},
$$

а потому при суммировании величин $\left|\partial S_{i}(G, f)\right|$ каждая вершина $u$ считается ровно

$$
\max \{f(v): v \in V, v \cong u\}-f(u)
$$

раз.

Поскольку нумерация $f$ произвольна, переходя к минимуму по всевозможным нумерациям в равенстве (1), получаем, что

$$
p^{*}(G)=\mathrm{vs}_{\mathrm{sum}}(G) \text {. }
$$

Теорема доказана.

Из этого утверждения и результатов работы [4] немедленно вытекает ряд следствий, полученных независимо китайскими математиками в [7].

Известно (см. [6]), что для любого графа величина вершинного разделения ровно на единицу больше так называемои интервальной ширины графа. Поэтому при изучении суммарной величины вершинного разделения возникает естественный вопрос: нельзя ли охарактеризовать этот инвариант с помощью графов интервалов? Оказалось, что ответ на этот вопрос положителен. Для того, чтобы сформулировать его (в переписанном для профиля виде), напомним, что граф называется графом интервалов, если его вершинам можно поставить в соответствие интервалы вещественной прямой таким образом, что две вершины являются смежными тогда и только тогда, когда соответствующие им интервалы пересекаются.

Следствие 1. Профиль графа $G$ совпадает с иислом ребер графа интервалов, содержащего граф $G$ в качестве подграфа и имеющего минимально возможное число ребер.

Из этого следствия вытекает утверждение о сложности вычисления профиля. В списке NP-полных задач, приведенном в [8], присутствует следующая задача распознавания: 
для заданного графа $G=(V, E)$ и неотрицательного целого числа $k$ выяснить, существует ли граф интервалов $G^{\prime}$ такой, что

$$
V\left(G^{\prime}\right)=V(G), \quad E(G) \subseteq E\left(G^{\prime}\right), \quad\left|E\left(G^{\prime}\right) \backslash E(G)\right| \leqslant k .
$$

Эта задача является NP-полной даже для реберных графов. Нетрудно видеть; что эта задача полиномиально преобразуется к нашей, и из следствия 1 вытекает следующее утверждение.

Следствие 2. Для заданного графа $G=(V, E)$ и положительного челого числа $k$ задача распознавания, верно ли, что $p(G) \leqslant k$, является $N P$-полной для реберных графов.

\section{4. Оценки профиля графов}

В этом разделе будут даны некоторые оценки профиля графов, полученные с помощью теоремы 1.

Предложение 1. Пусть $G-$ граф с $n$ вершинами и $m$ ребрами. Тогда

$$
m \leqslant p(G) \leqslant \frac{1}{2} n(n-1),
$$

причем $p(G)=m$ тогда и только тогда, когда $G$ - граф интервалов, $и p(G)=$ $n(n-1) / 2$ в том и толъко том случае, когда $G$ - полный граф.

Эта оценка сразу вытекает из следствия 1.

Оценим теперь профиль графа с помощью величины вершинного разделения.

Предложение 2. Пусть $G-$ граф с $n$ вериинами. Тогда

$$
\frac{1}{2} \operatorname{vs}(G)(\operatorname{vs}(G)+1) \leqslant p(G) \leqslant \frac{1}{2}(2 n+1-\operatorname{vs}(G)) \operatorname{vs}(G) .
$$

Если, кроме того, известно, что граф $G$ связен, то

$$
\frac{1}{2} \operatorname{vs}(G)(\operatorname{vs}(G)-1)+n-1 \leqslant p(G) \leqslant \frac{1}{2}(2 n+1-\operatorname{vs}(G)) \operatorname{vs}(G) .
$$

Доказательство. Положим $k=\mathrm{vs}(G)$. Пусть также $f-$ нумерация вершин графа $G$ такая, что

$$
p(G)=\operatorname{vs}_{\mathrm{sum}}(G)=\sum_{i=1}^{n-1}\left|\partial S_{i}(G, f)\right| .
$$

Нетрудно видеть, что найдутся такие

$$
i_{1}, i_{2}, \ldots, i_{k} \in\{1, \ldots, n-1\}, \quad i_{1}<i_{2}<\ldots<i_{k},
$$

для которых $\left|\partial S_{i_{j}}(G, f)\right|=j$ при $j=1, \ldots, k$. Если же $j \neq i_{1}, i_{2}, \ldots, i_{k}$, то $0 \leqslant$ $\left|\partial S_{j}(G, f)\right| \leqslant k$. Отсюда следует, что

$$
1+2+\ldots+k \leqslant \sum_{i=1}^{n-1}\left|\partial S_{i}(G, f)\right| \leqslant 1+2+\ldots+k+(n-k) k .
$$


Осталось заметить, что

$$
\begin{aligned}
1+2+\ldots+k & =k(k+1) / 2 \\
1+2+\ldots+k+(n-k) k & =(2 n+1-k) k / 2 .
\end{aligned}
$$

Второе неравенство доказывается аналогично. Достаточно заметить, что при $j \neq i_{1}, i_{2}, \ldots, i_{k}$ выполнено неравенство

$$
1 \leqslant\left|\partial S_{j}(G, f)\right| \leqslant k .
$$

\section{5. Профиль соединения графов}

Для большинства известных операций над графами удается получить лишь довольжо грубые оценки, связывающие характеристики исходных графов и профиль графа, полученного в результате операции. Однако для одной операции удается получить точное выражение. Это операция соединения графов. Отметим, что похожее выражение получается для величины вершинного разделения и поискового числа графов (см. [9]).

Пусть $G_{1}, G_{2}$ - графы, $V\left(G_{1}\right) \cap V\left(G_{2}\right)=\varnothing$. Соединением графов $G_{1}$ и $G_{2}$ (см. [10]) называется граф с множеством вершин $V\left(G_{1}\right) \cup V\left(G_{2}\right)$ и множеством ребер

$$
E\left(G_{1}\right) \cup E\left(G_{2}\right) \cup\left\{(u, v): u \in V\left(G_{1}\right), v \in V\left(G_{2}\right)\right\} .
$$

Мы будем обозначать соединение графов $G_{1}$ и $G_{2}$ через $G_{1}+G_{2}$.

Теорема 2. Пусть $G_{1}, G_{2}$ - графы,

$$
V\left(G_{1}\right) \cap V\left(G_{2}\right)=\varnothing, \quad\left|V\left(G_{1}\right)\right|=n_{1}, \quad\left|V\left(G_{2}\right)\right|=n_{2} .
$$

Тогда

$$
p\left(G_{1}+G_{2}\right)=\min \left\{n_{1}\left(n_{1}-1\right) / 2+p\left(G_{2}\right), n_{2}\left(n_{2}-1\right) / 2+p\left(G_{1}\right)\right\}+n_{1} n_{2} .
$$

Доказательство. Пусть $f$ - оптимальная нумерация вершин графа $G_{1}+G_{2}$, то есть такая нумерация, что

$$
p\left(G_{1}+G_{2}\right)=\operatorname{vs}_{\mathrm{sum}}\left(G_{1}+G_{2}\right)=\sum_{i=1}^{n_{1}+n_{2}-1}\left|\partial S_{i}\left(G_{1}+G_{2}, f\right)\right|
$$

Обозначим $k$ наименьшее положительное целое число, для которого либо $V\left(G_{1}\right) \subseteq$ $S_{k}\left(G_{1}+G_{2}, f\right)$, либо $V\left(G_{2}\right) \subseteq S_{k}\left(G_{1}+G_{2}, f\right)$. Пусть для определенности

$$
V\left(G_{1}\right) \subseteq S_{k}\left(G_{1}+G_{2}, f\right)
$$

Ясно, что $k \geqslant n_{1}$. Введем в рассмотрение нумерацию $g$ вершин графа $G_{2}$ такую, что если $u, v \in V\left(G_{2}\right)$, то $g(u)<g(v)$ тогда и только тогда, когда $f(u)<f(v)$. Иными словами, при нумерации $g$ вершины графа $G_{2}$ нумеруются в той же последовательности, что и при нумерации $f$. 
Из определения соединения графов вытекает, что

$$
\left|\partial S_{i}\left(G_{1}+G_{2}, f\right)\right|=i, \quad i=1, \ldots, k-1,
$$

а при $i=k, \ldots, n_{1}+n_{2}-1$

$$
\left|\partial S_{i}\left(G_{1}+G_{2}, f\right)\right|=n_{1}+\left|\partial S_{i-n_{1}}\left(G_{2}, g\right)\right|
$$

(предполагается, что $\left.S_{0}\left(G_{2}, g\right)=\varnothing\right)$. Заметим также, что $\left|\partial S_{i}\left(G_{2}, g\right)\right| \leqslant i$ для любого $i=1, \ldots, n_{2}$. Объединяя эти равенства и неравенства, получаем, что

$$
\begin{aligned}
p\left(G_{1}+G_{2}\right) & =\sum_{i=1}^{n_{1}+n_{2}-1}\left|\partial S_{i}\left(G_{1}+G_{2}, f\right)\right|=\sum_{i=1}^{k-1} i+\sum_{i=k}^{n_{1}+n_{2}-1}\left(n_{1}+\left|\partial S_{i-n_{1}}\left(G_{2}, g\right)\right|\right) \\
& =\sum_{i=1}^{n_{1}} i+\sum_{i=1}^{k-n_{1}-1} i+\sum_{i=k}^{n_{1}+n_{2}-1}\left|\partial S_{i-n_{1}}\left(G_{2}, g\right)\right|+n_{1}\left(n_{2}-1\right) \\
& =\frac{1}{2} n_{1}\left(n_{1}-1\right)+\sum_{i=1}^{k-n_{1}-1} i+\sum_{i=k-n_{1}}^{n_{2}-1}\left|\partial S_{i}\left(G_{2}, g\right)\right|+n_{1} n_{2} \\
& \geqslant \frac{1}{2} n_{1}\left(n_{1}-1\right)+\sum_{i=1}^{n_{2}-1}\left|\partial S_{i}\left(G_{2}, g\right)\right|+n_{1} n_{2} \geqslant \frac{1}{2} n_{1}\left(n_{1}-1\right)+p\left(G_{2}\right)+n_{1} n_{2} .
\end{aligned}
$$

Таким образом,

$$
p\left(G_{1}+G_{2}\right) \geqslant \min \left\{n_{1}\left(n_{1}-1\right) / 2+p\left(G_{2}\right), n_{2}\left(n_{2}-1\right) / 2+p\left(G_{1}\right)\right\}+n_{1} n_{2} .
$$

Докажем теперь, что выполнено обратное неравенство. Пусть $f$ - произвольная нумерация вершин графа $G_{1}$, а $g$ - оптимальная нумерация вершин графа $G_{2}$, то есть

$$
p\left(G_{2}\right)=\sum_{i=1}^{n_{2}-1}\left|\partial S_{i}\left(G_{2}, g\right)\right| .
$$

Введем в рассмотрение нумерацию $h$ вершин графа $G_{1}+G_{2}$, построенную следующим образом:

$$
h(v)= \begin{cases}f(v), & \text { если } v \in V\left(G_{1}\right), \\ n_{1}+g(v), & \text { если } v \in V\left(G_{2}\right) .\end{cases}
$$

Нетрудно видеть, что при $i=1, \ldots, n_{1}$

$$
\left|\partial S_{i}\left(G_{1}+G_{2}, h\right)\right|=i,
$$

а при $i=n_{1}+1, \ldots, n_{1}+n_{2}-1$

$$
\left|\partial S_{i}\left(G_{1}+G_{2}, h\right)\right|=n_{1}+\left|\partial S_{i-n_{1}}\left(G_{2}, g\right)\right| \text {. }
$$

Получаем, что

$$
\begin{aligned}
p\left(G_{1}+G_{2}\right) & \leqslant \sum_{i=1}^{n_{1}+n_{2}-1}\left|\partial S_{i}\left(G_{1}+G_{2}, h\right)\right|=\sum_{i=1}^{n_{1}} i+\sum_{i=n_{1}+1}^{n_{1}+n_{2}-1}\left(n_{1}+\left|\partial S_{i-n_{1}}\left(G_{2}, g\right)\right|\right) \\
& =\frac{1}{2} n_{1}\left(n_{1}+1\right)+n_{1}\left(n_{2}-1\right)+\sum_{i=1}^{n_{2}-1}\left|\partial S_{i}\left(G_{2}, g\right)\right| \\
& =\frac{1}{2} n_{1}\left(n_{1}-1\right)+p\left(G_{2}\right)+n_{1} n_{2} .
\end{aligned}
$$


Аналогично доказывается, что

$$
p\left(G_{1}+G_{2}\right) \leqslant n_{2}\left(n_{2}-1\right) / 2+p\left(G_{1}\right)+n_{1} n_{2}
$$

Отсюда следует неравенство

$$
p\left(G_{1}+G_{2}\right) \leqslant \min \left\{n_{1}\left(n_{1}-1\right) / 2+p\left(G_{2}\right), n_{2}\left(n_{2}-1\right) / 2+p\left(G_{1}\right)\right\}+n_{1} n_{2} .
$$

Теорема доказана.

Рассмотрим в качестве примера полный двудольный граф $K_{m, n}$. Ясно, что $K_{m, n}=E_{m}+E_{n}$, где через $E_{k}$ обозначается пустой граф (то есть граф без ребер), имеющий $k$ вершин. Очевидно, что $p\left(E_{k}\right)=0$ для любого $k$. Предполагая, что $m \leqslant n$, получаем $p\left(K_{m, n}\right)=m(m-1) / 2+m n$.

\section{Список литературы}

1. Chinn P. Z., Chvatalova J., Dewdeny A. K., Gibbs N. E. The bandwidth problem for graphs and matrices - a survey. J. Graph Theory (1982) 6, 223-254.

2. Bienstock D. Graph searching, path-width, tree-width and related problems (a survey) DIMACS ser. in Discrete Math. Theor. Comp. Sci. (1991) 5, 33-49.

3. Möhring R. H. Graph problems related to gate matrix layout and PLA folding. Computional Graph Theory, Computing Suppl. (1990) 7, 17-51.

4. Головач П. А. Суммарная величина вершинного разделения графов. Дискретная математика (1997) 9, №4, 86-91.

5. Ellis J. A., Sudborough I. H.,Turner J. S. Graph separation and search number. In: Proc. Allerton Conf. on Communication, Control and Computing. 1983, pp. 224-233.

6. Kirousis L. M., Papadimitriou C. H. Interval graph and searching. Discrete Math. (1985) 55, 181-184.

7. Lin Y. X., Yuan S. S. Profile minimization problem for matrices and graphs. Acta Math. Appl. Sinica (1994) 10, №1, 107-112.

8. Гэри М., Джонсон Д. Вычислителъные машины и труднорешаемые задачи. Мир, Москва, 1982.

9. Головач П. А. Вершинно-поисковое и поисковое число соединения графов. Вестник ЛГУ. Сер. 1 (1990) №2.

10. Харари Ф. Теория графов. Мир, Москва, 1973.

Статья поступила 09.02.1996. 